\title{
DEVELOPMENT OF A REAL TIME MONITORING AND ADAPTIVE CONTROL SYSTEM FOR LASER FLAME CUTTING
}

1203

\author{
J. R. Duflou ${ }^{1}$, E. Fallahi Sichani ${ }^{1}$, J. De Keuster ${ }^{1}$, J.-P. Kruth ${ }^{1}$ \\ ${ }^{1}$ Dept. of Mechanical Engineering, Katholieke Universitieit Leuven, Celestijnenlaan 300B, B-3001 \\ Heverlee (Leuven), Belgium
}

\begin{abstract}
A real-time, adaptive control and optimization system for laser flame cutting of thick plates of mild steel has been developed. The proposed system consists of two subsystems: a process monitoring system and a control and optimization system. The first subsystem aims at the on-line observation of the process status and corresponding cut quality. Since the different cut quality characteristics (e.g. cutting edge striations or dross) cannot be measured directly, the proposed system is based on so-called sensing parameters, which are easily-observable physical parameters that correlate well with the quality characteristics of the cut surface. The applicability of different optical sensors (photodiodes and a NIR-camera) has been investigated. The most optimal configuration of the process monitoring system is presented, including an overview of the selected set of sensing parameters.
\end{abstract}

The second subsystem, the real-time control and optimization system, supports the adaptation of the process parameters, based on the cut quality information obtained from the process monitoring system. A suitable hardware configuration for the real-time control and optimization is presented, starting from the original platform (i.e. an industrial 2D laser cutting machine). A generic expert strategy, has been designed for the control and optimization purpose. Using the developed experimental platform, the performance of the expert system was verified and optimized for different material-thickness combinations.

The obtained results demonstrate the effectiveness of the chosen approach in terms of increased autonomy, productivity, and efficiency of the process, as well as elimination of the need for manual quality control and the possibility to automatically generate quality reports.

Keywords: laser cutting, monitoring, adaptive control, optimization

\section{Introduction}

In recent years an expansion of the laser cutting range towards higher plate thickness could be observed. Quality assurance however becomes less obvious when processing plates of $20-25 \mathrm{~mm}$ and beyond: the process window becomes very narrow and limited disturbances in the surface quality (e.g. corrosion marks or sprayed on labels) or preheating conditions can be sufficient to cause severe quality deterioration and even loss of cut for a given set of process parameters. In this context, in order to avoid major scrap fractions, one has to call on appropriate real time monitoring and adaptive control systems. Today, however, no such systems are commercially available.

This paper focuses on the development and validation of such a monitoring and control system, suitable for the supervision of $\mathrm{CO}_{2}$ flame cutting in the multi-kW range. After a short state of the art in the remainder of this introductory section, the architecture of the chosen hardware configuration is documented (Section 2). The identified sensing parameters and corresponding quality parameters are reported in Section 3, while the architecture and the functionality of the derived adaptive control system are summarized in Section 4 and illustrated by means of a number of representative cases.

Several authors reported contributions to the development of monitoring and control systems. In most cases this research was limited to the integration of photodiodes and, to a lesser extent, pyrometers. The application of camera systems, and specifically of thermal cameras, for monitoring the laser cutting process has been reported in a limited number of research contributions only.

Jorgensen [1] monitored the laser cutting process by means of a Si-photodiode, a CCD camera and a pyrometer in two different coaxial set-ups. The mean value of the photodiode signal proved to correlate 
well with dross formation, whereas the variance could be correlated to the roughness of the cut edge. Moreover the striation frequency could be monitored using frequency analysis techniques. Although the results obtained from the photodiodes were most promising, the CCD images proved to also provide useful information: the mean pixel value of the recorded image proved to correlate well with dross formation, whereas the variance could be correlated to the roughness of the cut edge. Based on the images, the cut kerf width could also be measured.

Leidinger [2] used a $\mathrm{Si}$ photodiode to monitor the laser cutting process in different setups and came to similar conclusions as Jorgensen. It was also shortly mentioned that quality problems like plasma formation could be observed.

Chatwin et al. [3, 4, 5] reported the development of a knowledge-based adaptive control system for laser cutting based on a photodiode system and a CCDcamera system that captures the spark cone at the bottom of the plate. For both set-ups an off-axis setup was selected. The photodiode was used to measure the temperature/irradiance, emitted by the cut front. The most important information about the process status and corresponding cut quality was however obtained from the CCD images. During the interpretation of these images, the spark cone was divided into an inner dense and outer sparse cone. The authors reported that a bad cut quality was typically characterized by a larger, more diffuse spark cone. Image correlation techniques were applied for comparing ideal spark cone images with the observed spark cone.

Decker et al. [6] developed a collinear photodiode set-up using a partially transmitting mirror. The authors decided to move to this solution, because of the disadvantages linked to a camera set-up underneath the workpiece with respect to a flying optics architecture. The presented results are similar to those of Jorgensen and Leidinger. The authors confirmed the earlier reported correlations between the variance and cut roughness and the mean value and dross attachment. In order to improve the robustness of the monitoring system for control purposes, a variant based on a toric mirror and four photodiodes was developed. However the system was found to fail as a result of small changes in process parameters (like beam parameter settings) or when preheating occurred.

Kaplan et al. [7] sensed the thermal emissions from the cutting front using a selective $\mathrm{ZnSe}$ mirror and $\mathrm{Si}$ photodiode. The roughness proved to be proportional to the detected striation period, whereas the occurrence of a burning defect could be detected by a quality factor combining the mean and variance of the photodiode signal. The authors also suggested that the information obtained from the monitoring system could be used to modulate the laser power using a fuzzy control system. This was however not implemented.

Sforza et al. [8] used an IR monitoring system consisting of a Si-photodiode, a bandpass filter (800$1000 \mathrm{~nm}$ ) and a fibre-optic guidance system for monitoring the laser cutting process. The authors aimed at control of the striation frequency using laser power modulation. More specifically the laser was modulated at a frequency higher than the natural one. As a result, the roughness was considerably reduced, which could clearly be observed by a reduced variation of the IR signal.

Kaebernick et al. [9] developed an adaptive control system for laser cutting in order to improve the surface roughness by influencing the striation frequency. The used monitoring system consisted of a photodiode, installed behind a $\mathrm{ZnSe}$ dichroic mirror. The authors showed that the roughness decreases quasi linearly with increasing striation frequency. To extract the striation frequency from the photodiode signal, the Welch method (i.e. an estimation method for the spectral content of the signal) was used.

At the Fraunhofer Institute for Laser Technology, Poprawe et al. [10,11] developed a universal coaxial process control system, a modular system that can be equipped with different sensors, like CCD-cameras and photodiodes, for monitoring different laser manufacturing processes, like welding, cutting, marking, etc. Results concerning the integration of a high speed CCD camera in this universal system were reported. For use in a $\mathrm{CO}_{2}$ laser system an optical setup was elaborated using a $2 \mathrm{~mm}$ pinhole mirror. The investigations with regard to cutting were limited to the fusion cutting process. The cut kerf and effects like cutting interruption or instabilities leading to dross formation could be visualized.

Tönshoff et al. [12] developed monitoring systems for respectively $\mathrm{CO}_{2}$ laser cutting and $\mathrm{Nd}$ :YAG laser welding. The aim of the research was, by integrating appropriate sensors, not only to detect faults and defects, but also to classify sources of defects. In the $\mathrm{CO}_{2}$ laser cutting head, the following sensors were integrated:

- An eight-quadrant thermopile for identifying misalignment, power loss or beam distortion 
- A photodiode-based process monitoring sensor, combined with a diffractive mirror

- A microphone with a slightly non-linear frequency response

Different types of machine faults were simulated. The reported results were however rather qualitative. It was proven that both the acoustic and photodiode signal are very irregular when the cut is incomplete. Stable process conditions are characterized by a stable and low signal level. Finally it was stated that the gas delivery can best be monitored by the microphone.

Haferkamp et al. [13] developed an on-line thermographic system, based on a CCD-camera, for the control of Nd:YAG laser cutting of sheet metal measuring the temperature field emitted from the processing zone. Mainly the fusion cutting process was considered. Strong correlations between thermal image parameters and the cutting quality and machining conditions were reported. The set-up made use of a dichroic mirror for the out-coupling of the process light: the Nd:YAG light was reflected, whereas the mirror was transparent for the heat radiation emitted by the process. Maladjustment of the laser beam relative to the gas jet could be detected by an asymmetric temperature distribution. Dross attachment resulted in an enlarged temperature field and higher values at the edges. The cutting width could also be correlated to the distance between the two peaks of the kidney-like temperature field. Monitoring of the breakthrough for piercing was reported. Differences in beam power distribution and surface conditions could also be observed. It was also suggested that by splitting the temperature image into the three colour channels, control of the laser cutting process might be possible using threshold values. This was however not discussed in further detail.

In previous publications the authors of this paper reported on the use of acoustic and photodiode based systems for process monitoring of higher power $\mathrm{CO}_{2}$ flame cutting $[14,15,16]$. In line with observations made by the researchers referred to above, the authors concluded that photodiodes can provide useful information for quality assurance purposes with a very limited time delay. The spatial information offered by the use of camera systems in general, and of an IR camera in specific, proved however to offer more refined process analysis capabilities that were found to be beneficial for the development of a robust and reliable adaptive control system. In the remainder of this paper, findings in this respect are reported and the hardware configuration used for this research is described. The performance of the derived control system is illustrated by means of representative cases.

\section{Experimental setup}

The experimental setup described in this paper was based on a 2D laser cutting machine with flying optics architecture, a $6 \mathrm{~kW} \mathrm{CO}$ laser source $\left(\mathrm{M}^{2}=\right.$ 4.24) and a Fanuc controller.

Although camera placement below the cutting table could offer specific advantages in terms of spark cone pattern observation $[3,4,5]$, the unfriendly environment (risk for damaging the camera with dust and molten material expulsion) and the need for an additional camera positioning system formed counter indications for this option. A coaxial setup with $\mathrm{ZnSe}$ focusing lens was preferred in order to have full benefit of the protective screen offered by the lens and an assured, position independent camera alignment.

Different optical configurations were investigated, finally resulting in a setup with a dichroic mirror transmitting the laser beam and reflecting light in the near infrared (NIR) spectrum towards the camera setup (Figure 1). Additional optical elements, such as a focusing lens, optical filters and a beam-splitter folding mirror were included to assure optimal process light transmittal to both a camera and a photodiode sensor system (not shown on Fig. 1).

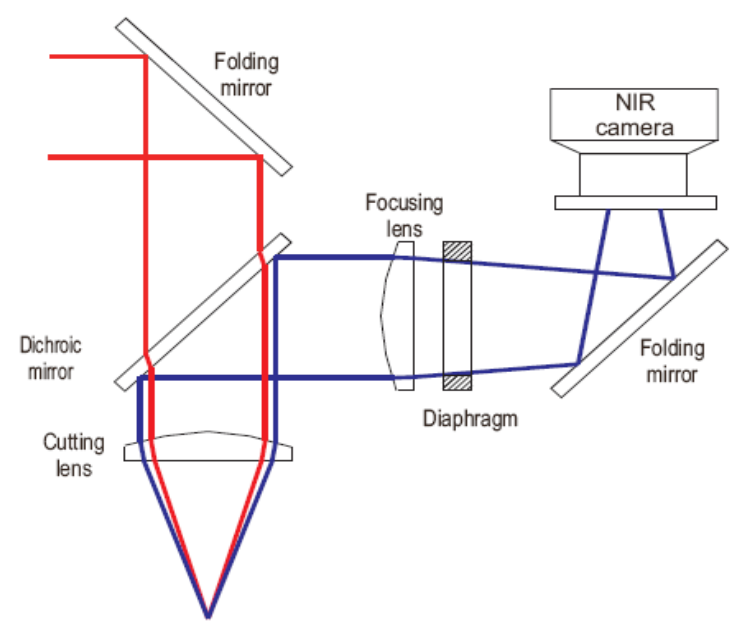

Figure 1: Optical setup for near-infrared camera monitoring system

Taking the melt temperature of steel and the laws of Planck and Wien into account, it can easily be verified that the peak wavelength of the emission spectrum is situated around $1.7 \mu \mathrm{m}$. Also chromatic 
aberration of the cutting lens was taken into account to optimize the spectral sensitivity of the camera system. The focus point variation in function of the wavelength was studied and is represented in Fig. 2. It can clearly be observed that the focal point position varies more at shorter wavelengths. Consequence is that the depth of focus in the visual spectrum would be low, while in the infrared region the variation of the focal point is much smaller. Based on this knowledge, an NIR sensor type was chosen, more specifically a camera based on InGaAs technology and sensitive in the NIR spectrum, ranging from 0.9 to $1.7 \mu \mathrm{m}$.

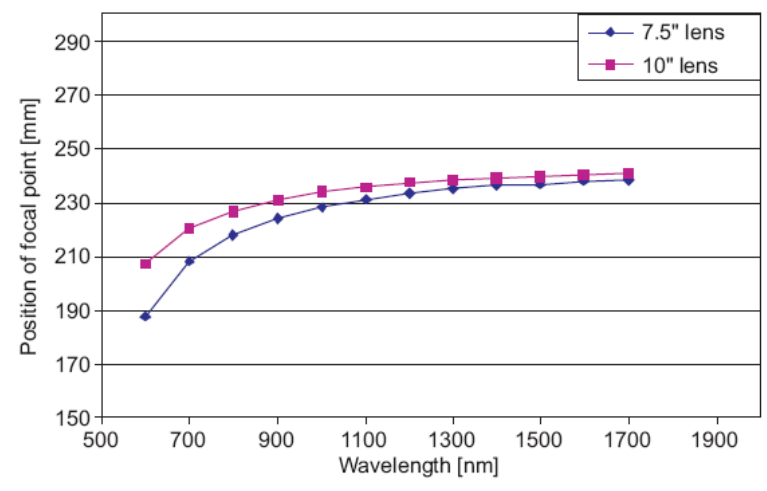

Figure 2: The position of the focal point in function of the wavelength for $\mathrm{f}_{\text {camera lens }}=250 \mathrm{~mm}$

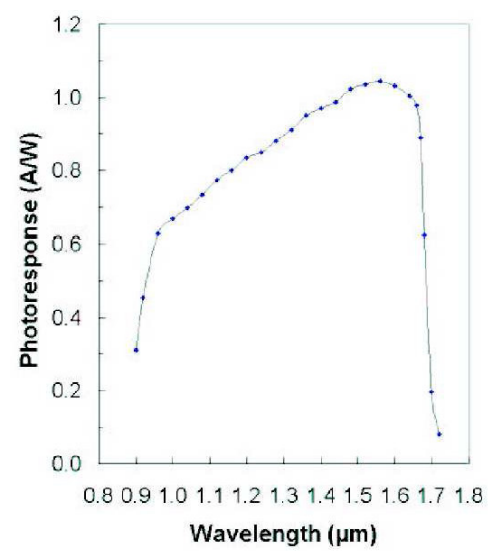

Figure 3: The spectral sensitivity of the selected camera detector

The spectral sensitivity of the camera detector is illustrated in Fig. 3. The resolution of the camera is $256 \times 320$ pixels (with a pixel size of $30 \times 30 \mu \mathrm{m}^{2}$ ), which is large enough for visualizing the entire process front and its direct neighbourhood. Camera settings like integration time were optimised in order to obtain a clear and unsaturated image at all cutting conditions.

\section{Quality characteristics and sensing parameters}

Based on consultation of relevant standards $[17,18,19]$ and discussion with industrial partners, the following cut quality characteristics were selected for monitoring purposes:

- Dross attachment: quantified by means of the height of the dross measured after cutting (by a non-contact sensor) or height of the re-attachment zone ('grey zone' after dross removal)

- Roughness: quantified by means of $\mathrm{R}_{\mathrm{z}}$, measured at a height as specified in [17] and [18], with a cut-off wavelength of $2.5 \mathrm{~mm}$ (measured by a non-contact profilometer)

- Drag of the striations: quantified as the distance between the start of a striation and the end point of the striation into which it passes, orthogonally projected to the plate surface (measured by optical microscope)

- Occurrence of burning defects: quantified as the average number of observed burning defects per length unit

- Squareness of the cut edge: quantified as per [17], measured by means of a tactile probe

- Cut width: idem

Sensing parameters or combinations thereof (i.e. performance indices) have been identified, that show good correlation with one or more of these specific cut quality characteristic(s), as illustrated in the next paragraphs. Extensively tested material-thickness combinations for this purpose were ST52-3 $15 \mathrm{~mm}$, ST52-LQ $20 \mathrm{~mm}$ and HARDOX-400 25mm. Other material-thickness combinations were used for verification purposes.

Fig.4 shows NIR images that are recorded during the laser flame cutting of ST52-3 $15 \mathrm{~mm}$ with increasing speed (starting at the optimal cutting speed and going up to $120 \%$ ). From the resulting samples it can be clearly seen that, when increasing the cutting speed, the drag of the striations and the dross attachment increase significantly (see Table 1). This quality deterioration can clearly be recognized in the NIR images: the dimensions of the central hot zone increase significantly, particularly along the cutting direction. This can mainly be explained by the elongation (and slightly higher location) of the process front due to the lack of energy input. 


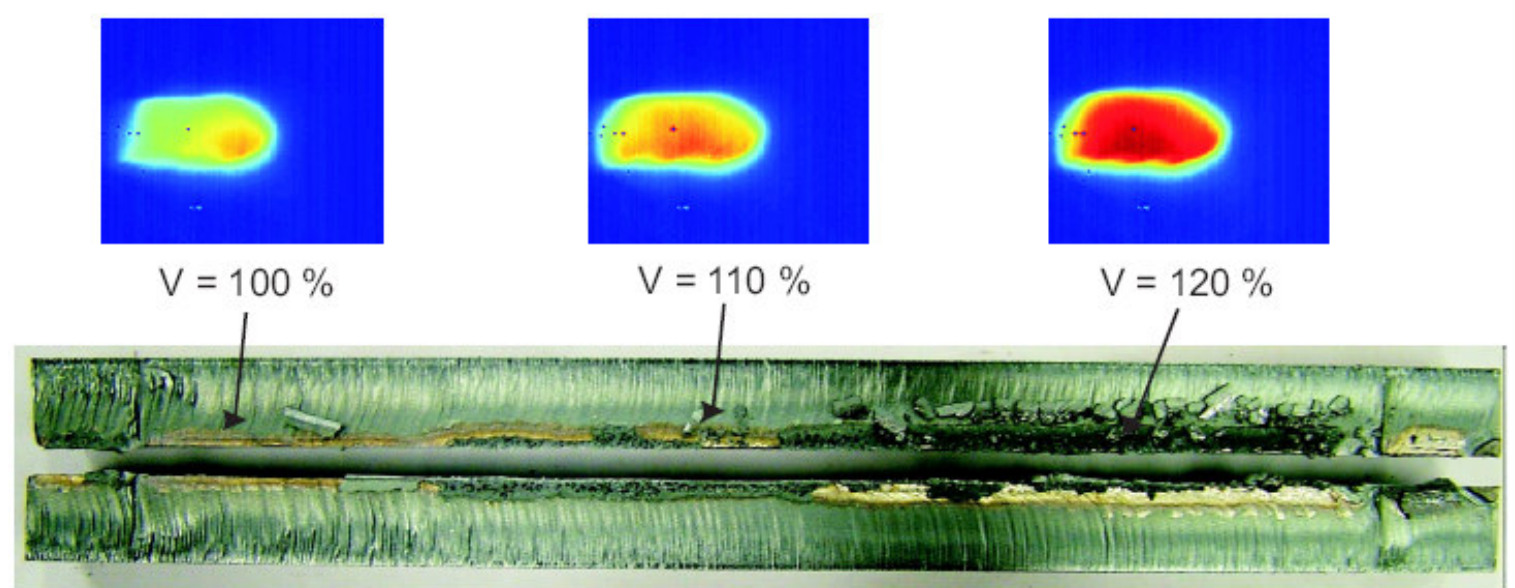

Figure 4: NIR images vs. cut quality for laser flame cutting ST52-3 $15 \mathrm{~mm}$ with increasing speed

Table 1: Quality parameters in the different cutting zones of Fig. 4

\begin{tabular}{|c|c|c|c|}
\hline Quality parameter & $\mathrm{V}=100 \%$ & $\mathrm{~V}=110 \%$ & $\mathrm{~V}=120 \%$ \\
\hline Drag of striations [mm] & 2.95 & 3.50 & 5.25 \\
\hline Grey zone (indicator for dross attachment) $[\mathrm{mm}]$ & 1.4 & 3.1 & 4.2 \\
\hline
\end{tabular}

When cutting is performed with decreasing cutting speed (e.g. from $100 \%$ to $60 \%$ ), the cut quality deterioration is different (see Fig. 5): the drag of the striations decreases and no dross attachment occurs, but the roughness, occurrence of burning defects and cut kerf width all increase. Indications of this quality deterioration can be clearly determined in the recorded NIR images. The length of the process zone becomes shorter and the intensity (related to the temperature) decreases significantly. This is the result of the surplus of process energy, leading to a straighter process front and corresponding, easier melt removal. When the cutting speed is reduced significantly, the heat input per unit length becomes too large and burning defects start to occur. The occurrence of a burning defect can easily be detected based on the NIR images: the width of the tail of the process front increases significantly, as illustrated in the NIR image corresponding to cutting at $\mathrm{V}=60 \%$. Similar observations could be made for all investigated material-thickness combinations. It can thus be concluded that clear distinction can be made between different types of quality deterioration for the flame cutting process.

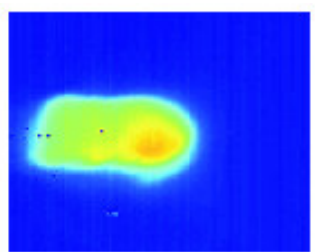

$V=100 \%$

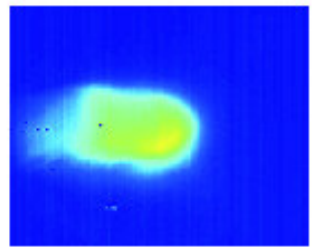

$\mathrm{V}=90 \%$

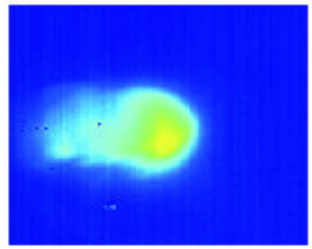

$\mathrm{V}=80 \%$

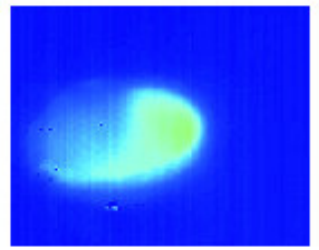

$V=70 \%$

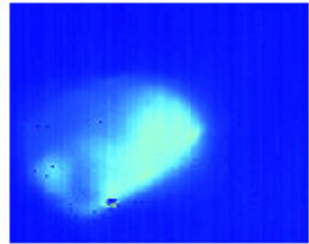

$V=60 \%$

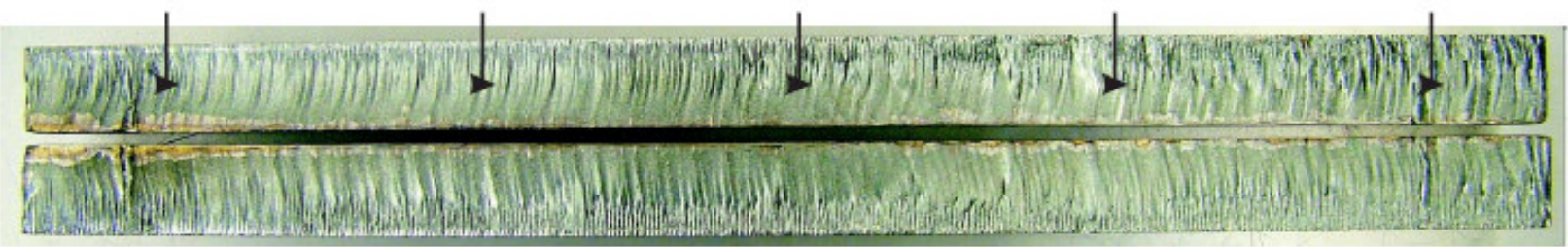

Figure 5: NIR images vs. cut quality for laser flame cutting ST52-3 $15 \mathrm{~mm}$ with decreasing speed 
Table 2: Overview of identified suitable sensing parameters

\begin{tabular}{|c|c|}
\hline Quality characteristic & Sensing parameters \\
\hline Dross attachment & $\begin{array}{l}\text { Length of the hot process zone }(\mathrm{ADU}>3000) \\
\text { Area of the hot process zone }(\mathrm{ADU}>3000)\end{array}$ \\
\hline Roughness & $\begin{array}{l}\text { (Variation of the) width of the circumscribed rectangle of the } \\
\text { global process zone (ADU }>1000)\end{array}$ \\
\hline Drag of striations & $\begin{array}{l}\text { Length of the hot process zone }(\mathrm{ADU}>3000) \\
\text { Area of the hot process zone }(\mathrm{ADU}>3000)\end{array}$ \\
\hline Occurrence of burning defects & $\begin{array}{l}\text { (Variation of the) width of the circumscribed rectangle of the } \\
\text { global process zone }(\text { ADU }>1000)\end{array}$ \\
\hline Cut width & Method based on the gradient field of the NIR image \\
\hline \multicolumn{2}{|c|}{ ADU: Analog-to-Digital Unit for an integration time of $25 \mathrm{~ms}$. } \\
\hline
\end{tabular}

All sensing parameters listed in this table can be applied for real time monitoring at a $40 \mathrm{~Hz}$ sampling frequency, except the cut width determination by means of the gradient method, which requires a relatively high processing time $(39 \mathrm{~ms}$ on the tested setup). This method is based on the determination of the gradient between neighbouring pixel values for every pixel in the window of interest (see Fig. 6) and the determination of the distance between the two extreme peak values in the direction orthogonal to the cutting direction. The cut width is mainly of importance for dimensional accuracy verification in order to assure dimensional tolerance compliance of the workpiece. Real time determination at a high frequency is therefore not required.

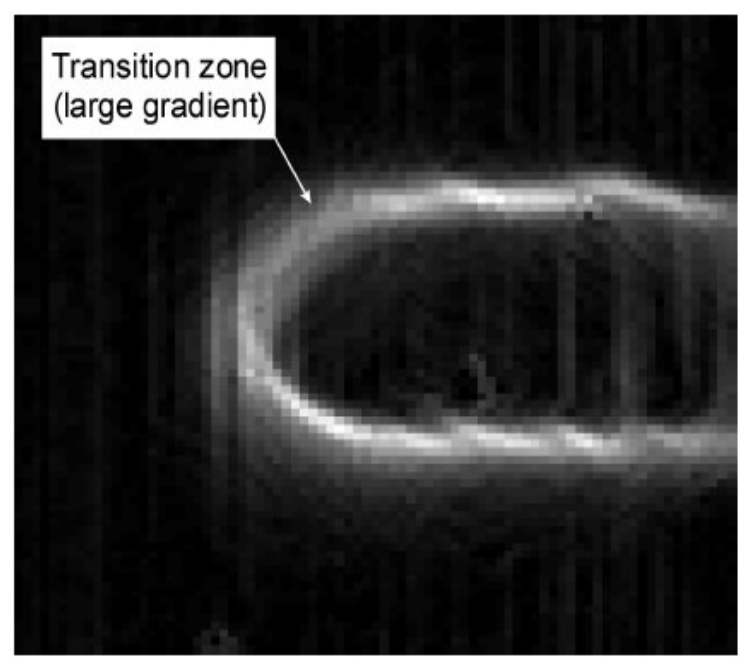

Figure 6: Gradient image of NIR image
Although useful NIR image sensing parameters could be determined for the dross, the roughness, the drag of striations, the occurrence of burning defects and the cut kerf width, no NIR image parameters could be identified that correlate well to the squareness of the cut edge. This lack of correlation can be explained by the fact that the NIR camera monitors the emission from a view point above the workpiece and therefore observes almost exclusively the upper part of the process zone. Furthermore the relatively low depth of focus (compared to the workpiece thickness) prevents explicit comparison of observations made at different depths in the kerf.

The conclusions drawn here have proven to be commonly valid for all tested material-thickness combinations. Nevertheless the actual relation between the NIR image parameters and cut quality varies slightly in function of the selected combination. An efficient test procedure was therefore worked out in order to allow calibration of the threshold levels for the different sensing parameters as used for control purposes (Section 4).

\section{Adaptive control system}

As control algorithm a range of possible strategies were investigated. Neural control, model-based control, Downhill Simplex optimization, fuzzy control, adaptive control constraint (ACC) and adaptive control optimization (ACO) strategies were explored. The adaptive control system was finally implemented as a fuzzy logic rule based system. 


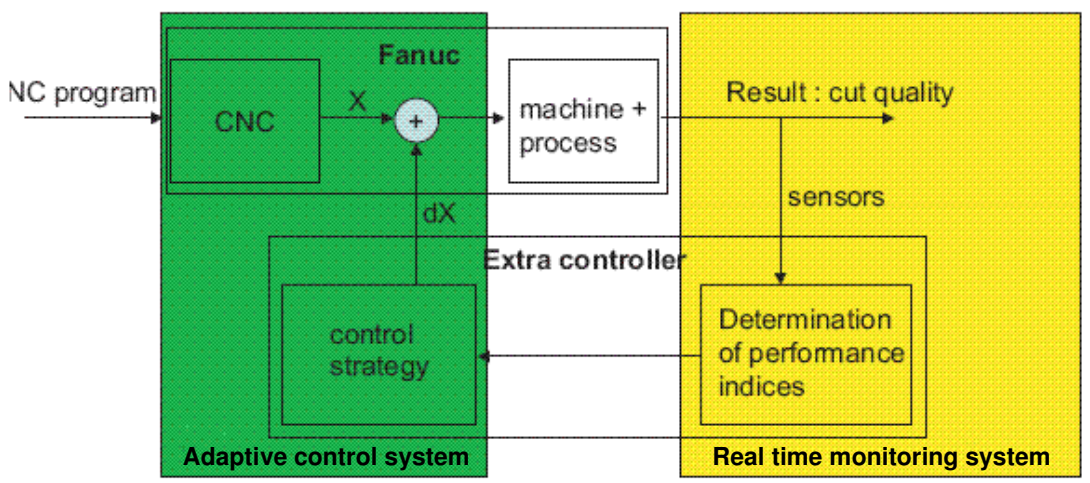

Figure 7: Overview scheme for the real time monitoring and control system

Based on the observed sensing parameter values, corresponding to the different quality parameters, and well-chosen threshold values, different quality classes are distinguished. Where quality deterioration is observed, appropriate corrective strategies are implemented by adjusting the most effective control parameter settings in an overwrite mode imposed by the adaptive control system to the CNC controller (see Fig. 7).

The adaptive control system has been implemented on an industrial PC platform with a real time Linux OS. The NIR camera input is organized by means of a USB interface. An additional data acquisition board facilitates the communication of this extra PC-based controller with the original Fanuc CNC system and enables complementary data acquisition, such as e.g. cutting direction information. An overwrite function is used to allow real time adjustment of the selected process control parameters. For this purpose the laser power $(\mathrm{P})$, cutting velocity $(\mathrm{V})$, assist gas pressure $(\mathrm{p})$ and duty cycle (DC) are used as control variables. The total response time corresponding to these control parameters (actuation + process response time) was found to be typically of order of magnitude of 100 to $150 \mathrm{~ms}$.

An optimization strategy was integrated in the adaptive control functionality with a maximized productivity as objective function. Where the cutting velocity can be increased without loss of quality, the process window is systematically explored for more productive settings.

The performance of the rule based adaptive control and optimization algorithms was tested in a wide range of situations. The following examples illustrate the effectiveness of the system. Fig. 8 documents the control and optimisation of a linear cutting operation in HARDOX-400 $25 \mathrm{~mm}$ starting from standard process settings. It can be observed that, except for some minor defect at the outset (due to the optimisation delay, i.e. an initial time period during which no adjustments are applied to the process control parameters in order to obtain a stable process status), the cut quality is satisfactory. The optimisation resulted in a cutting speed increase of approximately $21 \%$ (or $168 \mathrm{~mm} / \mathrm{min}$ ) as a result of an increase of the duty cycle and the gas pressure (respectively set at $75 \%$ and 0.5 bar in the standard cutting technology database).

The performance of the control and optimisation system has also been investigated for cases where the initial process settings significantly differ from the stable standard settings. Fig. 9 illustrates the control and optimisation of a linear cutting operation in ST52-3 $15 \mathrm{~mm}$, starting with a cutting velocity that is $20 \%$ higher than the standard setting. It can be noted that at the outset of the cut some drastic actions are required to correct the process status and corresponding cut quality. This is caused by the fact that in the lead-in zone, in which the laser cutting process still has to stabilise, the cutting velocity is too high with respect to the available energy input, leading to the detection of increased drag, increased risk of dross attachment (or even loss of cut).

Also for complex shaped workpieces with typical heat accumulation problems the algorithm proved effective.

Although robust and effective in process optimisation, the adaptive control system can not enlarge the process window as such. Where quality deterioration can not be prevented due to process limitations, the monitoring system can provide a systematic quality report indicating problematic cutting locations. This facilitates efficient quality inspection in case of manless production. 

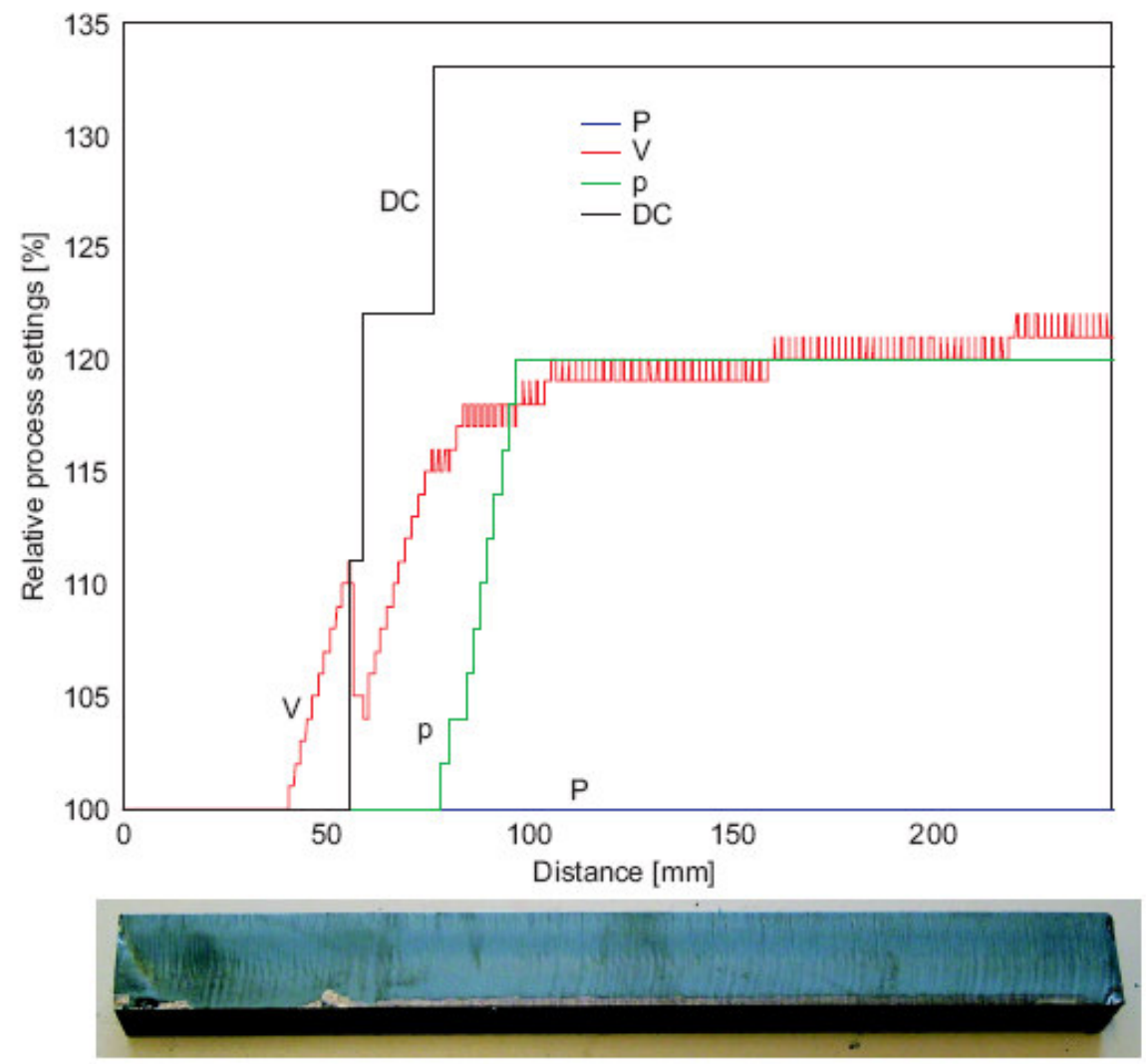

Figure 8: Control and optimisation of linear cutting of HARDOX-400 $25 \mathrm{~mm}$ starting from standard process settings

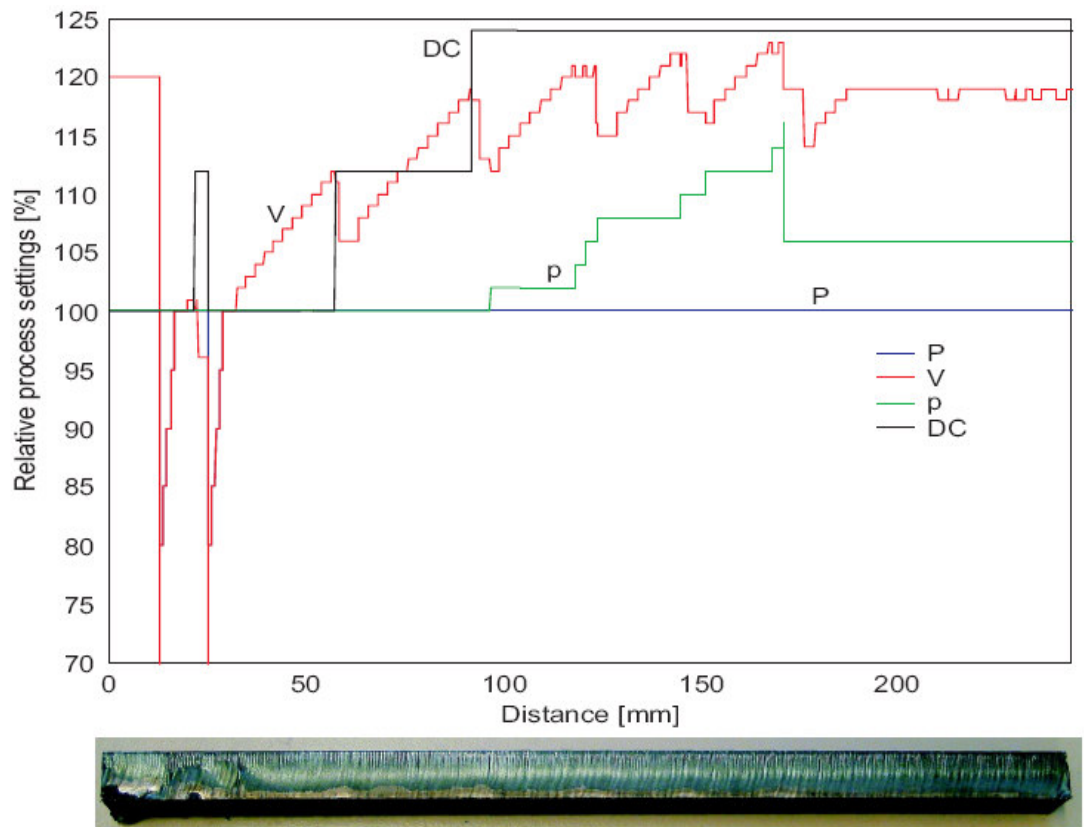

Figure 9: Control and optimisation of linear cutting of ST52-3 $15 \mathrm{~mm}$, starting from V=120 \% of $\mathrm{V}_{\text {standard }}$ 


\section{Conclusions}

It was experimentally demonstrated that a NIR camera based system can allow monitoring of the main quality parameters in $\mathrm{CO}_{2}$ flame laser cutting through well-chosen sensing parameters. While dross attachment, roughness, drag of striations and the occurrence of burning defects can be monitored in real time, the kerf width can be determined at a sufficient sample frequency to assure dimensional quality control in support of automatic dimensional tolerance verification. Only for the cut squareness no suitable sensing parameter for in process observation could be determined. This could be expected when considering that the tested monitoring system basically collects 2D information in a direction orthogonal to the observation plan required for squareness measurement. The developed real time monitoring system was integrated in an adaptive control system for automatic process quality assurance. The functional performance of this control system was illustrated by means of two representative demonstrative cases.

\section{References}

[1] Jorgensen H. (1990) Investigations of On-line Process Monitoring and Control in $\mathrm{CO}_{2}$ Laser Cutting. PhD thesis, Technical University of Denmark, Lyngby, Denmark.

[2] Leidinger D. (1995) In-process monitoring during $\mathrm{CO}_{2}$ laser cutting. Lasers in Engineering 4, 243-254.

[3] Huang M.Y. and Chatwin C.R. (1994) A knowledge-based adaptive control environment for an industrial laser cutting system. Optics and Lasers in Engineering 21, 273-295.

[4] Huang M.Y. and Chatwin C.R. (1994) Spark cone characterization for control of laser cutting. Lasers in Engineering 3, 125-140.

[5] Lim S.Y. and Chatwin C.R. (1994) Intelligent digital control of a laser cutting process. Lasers in Engineering 3, 99-112.

[6] Decker I., Heyn H., Martinen D., and Wohlfahrt H. (1997) Process monitoring in laser beam cutting on its way to industrial application, In Proceedings of the SPIE, volume 3097, pp 29-37.

[7] Kaplan A.F.H., Wangler O., and Schuöcker D. (1997) Laser cutting: Fundamentals of the periodic striations and their on-line detection, Lasers in Engineering 6, 103-126.

[8] Sforza P., de Blasiis D., Lombardo V., Santacesaria V., and Dell'Erba M. (1997) A threemodules sensor for $\mathrm{CO}_{2}$ laser welding and cutting processes, in Proceedings of the SPIE, Vol. 3097, 79107.

[9] Kaebernick H., Jeromin A., and Mathew P. (1998) Adaptive control for laser cutting using striation frequency analysis, in Annals of the CIRP 47, 137-140.

[10] Poprawe R. and Konig W. (2001) Modeling, monitoring and control in high quality laser cutting, in Annals of the CIRP 50, 137-140.

[11] Abels P., Kaierle S., Kratschz C., Poprawe R., and Schulz W. (1999) Universal coaxial process control system for laser materials processing, in Proceedings of the ICALEO 1999, Vol. 87, E99-108.

[12] Tönshoff H.K., Ostendorf A., Kral V., and Hillers O. (1999) Process and condition monitoring features incorporated in laser heads, in Proceedings of the ICALEO 1999, Vol. 87, E109-118.

[13] Haferkamp H., von Alvensleben F., von Busse A., Goede M., and Thurk O. (2000) Thermographic system for process monitoring of laser beam cutting, in Proceedings of the 8th international conference on sheet metal, 261-270.

[14] De Keuster J., Duflou J.R., Kruth J.-P. (2005) Monitoring of laser cutting by means of acoustic and photodiode sensors, in Proceedings of the 11th International conference on sheet metal, Erlangen, 809-816

[15] De Keuster J., Duflou J.R., Kruth J.-P. (2007) Real-time, adaptive control and optimization of highpower $\mathrm{CO}_{2}$ laser cutting using photodiodes, in Proceedings of LANE'07, 979-992

[16] De Keuster J., Duflou J. R., Kruth J.-P. (2007) Monitoring of high-power $\mathrm{CO}_{2}$ laser cutting by means of acoustic microphone and photodiodes, International Journal of Advanced Manufacturing Technology 35 (1-2), 115-126

[17] ISO 9013: Thermal cutting - Classification of thermal cuts - Geometrical product specification and quality tolerances 
[18] VDI 2906: Blatt 8, Quality of cut faces of (sheet) metal parts after cutting, blanking, trimming or piercing - Laser cutting

[19] DIN 2310: Thermal cutting - Part 30: Classification of thermal cuts, principles of process, quality and dimensional tolerances

\section{Authors autobiography}

- Joost R. Duflou holds master degrees in Architectural and Electro-mechanical Engineering and $\mathrm{a} \mathrm{PhD}$ in Engineering from the K.U.Leuven, Belgium. After a number of years of industrial experience, he joined the Asian Institute of Technology. Since 1997 he is active at the Mechanical Engineering Department of the K.U.Leuven, since 2006 as a tenure Professor. As chairholder of the LVD Chair on Sheet Metal Processing he leads a research group focussing on sheet metal oriented manufacturing processes, with special attention for laser cutting. He is an associate member of CIRP and has published over 200 international publications.

- Ehsan Fallahi Sichani holds master degree in Manufacturing and Production Engineering from Amirkabir University of Technology (Tehran Polytechnic), Iran. Since 2007 he has been conducting research on $\mathrm{CO}_{2}$ laser material processing as Ph.D. student at the Mechanical Engineering Department of the K.U.Leuven, Belgium.

- Johan De Keuster graduated as electromechanical engineer at the mechanical engineering department of the University of Leuven (K.U. Leuven), Belgium. After conducting research in the field of laser materials processing and more specifically highpower laser cutting, he obtained his Ph.D. degree with the dissertation, entitled "Development of an Adaptive, Real-Time Control and Optimisation System for High-Power $\mathrm{CO}_{2}$ Laser Cutting of Thick Plates". Now he works as project engineer for BASF, The Chemical Company.

- Jean-Pierre Kruth is Full Professor at the University of Leuven (K.U.Leuven), Belgium, where he is responsible for Production Engineering. His main research activities are: CAD/CAM/CAPP, production processes, metal cutting, non-traditional machining (EDM, laser machining, etc.), rapid prototyping \& manufacturing, dimensional metrology, quality control, reverse engineering. $\mathrm{He}$ is a/o Fellow Member of CIRP, Fellow of SME, Honorary member Romanian Society of Mech. Eng., Member BSMEE, IMEKO/BEMEKO and K.VIV, and founding board member of the companies Materialise (>1997), Metris and LayerWise. 